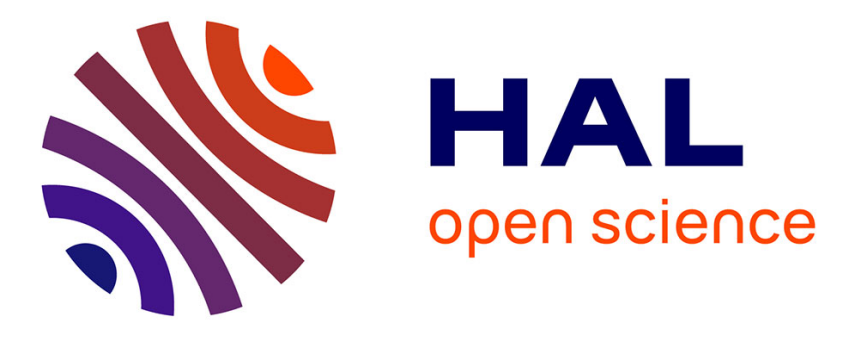

\title{
Filtering smooth altimetric signals using a Bayesian algorithm
}

Abderrahim Halimi, Gerald S. Buller, Steve Mclaughlin, Paul Honeine

\section{To cite this version:}

Abderrahim Halimi, Gerald S. Buller, Steve Mclaughlin, Paul Honeine. Filtering smooth altimetric signals using a Bayesian algorithm. Proc. 23rd European Conference on Signal Processing (EUSIPCO), 2016, Budapest, Hungary. pp.2385-2389, 10.1109/EUSIPCO.2016.7760676 hal-01965917

\section{HAL Id: hal-01965917 https://hal.science/hal-01965917}

Submitted on 27 Dec 2018

HAL is a multi-disciplinary open access archive for the deposit and dissemination of scientific research documents, whether they are published or not. The documents may come from teaching and research institutions in France or abroad, or from public or private research centers.
L'archive ouverte pluridisciplinaire HAL, est destinée au dépôt et à la diffusion de documents scientifiques de niveau recherche, publiés ou non, émanant des établissements d'enseignement et de recherche français ou étrangers, des laboratoires publics ou privés. 


\title{
FILTERING SMOOTH ALTIMETRIC SIGNALS USING A BAYESIAN ALGORITHM
}

\author{
Abderrahim Halimi $^{(1)}$, Gerald Buller ${ }^{(1)}$, Steve McLaughlin ${ }^{(1)}$ and Paul Honeine $^{(2)}$ \\ (1) Heriot-Watt University, School of Engineering and Physical Sciences, Edinburgh, U.K \\ ${ }^{(2)}$ Normandie Univ, UNIROUEN, UNIHAVRE, INSA Rouen, LITIS, Rouen, France.
}

\begin{abstract}
This paper presents a new Bayesian strategy for the estimation of smooth signals corrupted by Gaussian noise. The method assumes a smooth evolution of a succession of continuous signals that can have a numerical or an analytical expression with respect to some parameters. The Bayesian model proposed takes into account the Gaussian properties of the noise and the smooth evolution of the successive signals. In addition, a gamma Markov random field prior is assigned to the signal energies and to the noise variances to account for their known properties. The resulting posterior distribution is maximized using a fast coordinate descent algorithm whose parameters are updated by analytical expressions. The proposed algorithm is tested on satellite altimetric data demonstrating good denoising results on both synthetic and real signals. The proposed algorithm is also shown to improve the quality of the altimetric parameters when combined with a parameter estimation strategy.
\end{abstract}

Index Terms - Altimetry, Bayesian algorithm, coordinate descent algorithm, gamma Markov random fields

\section{INTRODUCTION}

A satellite altimeter is a nadir-viewing radar that emits regular pulses and records the travel time, the magnitude and the shape of each return signal after reflection on the Earth's surface. This reflected echo provides information about some physical parameters such as the range between the satellite and the observed scene (denoted by $\tau$ ), the significant wave height (denoted by SWH) and the wind speed (related to the signal's amplitude $P_{u}$ ). The oceanic altimetric signals can be described as a succession of continuous functions corrupted by noise [1-3] (see Fig. 1 (top) that shows a succession of 800 signals acquired by the Jason- 2 mission). Many recent studies and missions have focused on improving the quality of these signals by reducing the noise effect. This goal is achieved by amending the estimation algorithm to incorporate the known smooth properties of the altimetric parameters [4-6] or by operating on the observed signals to reduce their noise $[7,8]$. This paper adopts this second approach by

This work was supported by the EPSRC Grants EP/JO15180/1, EP/N003446/1, and EP/K015338/1 exploiting the smooth evolution of the successive altimetric signals. The main motivation for this choice is to propose a denoising algorithm that is independent from the parameter estimation algorithm, thus, it can be easily combined with any existing estimation algorithms [4-6,9] leading to an improvement in parameter estimation.
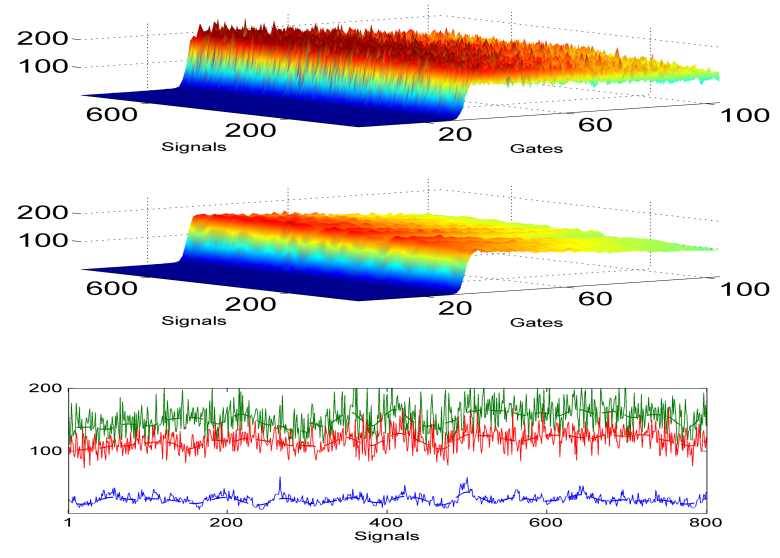

Fig. 1. (Top) Example of 800 noisy Jason-2 signals. (Middle) Filtered Jason-2 signals by the proposed SSE algorithm. (Bottom) Signal evolutions in gates $(30,50,90)$ (continuous lines) and their smooth approximation with SSE (dashed lines).

The first contribution of this paper is the development of a hierarchical Bayesian model to denoise a set of smooth signals. Each signal is assumed to be corrupted by additive, independent and non identically Gaussian noise. This noise model generalizes the independent and identically distributed (i.i.d.) Gaussian noise that is generally assumed when considering altimetric data $[9,10]$. A gamma Markov random field (GMRF) prior [11] is considered to account for the correlation between the noise variances and to better approximate the speckle noise. The signal energies are also assigned a GMRF prior to better approximate their continuity. Note that the proposed Bayesian hierarchy is generic in the sense that it does not assume a specific signal model. Indeed, the signal can be expressed by a numerical formula or by a given linear/nonlinear analytical function with respect to (w.r.t.) some parameters. 
The second contribution of this paper is the derivation of a denoising algorithm associated with the proposed hierarchical Bayesian model. The minimum mean square error (MMSE) and maximum a posteriori (MAP) estimators of the unknown signals/parameters cannot be easily computed from the obtained joint posterior. In this paper, the MAP estimator is evaluated by considering a coordinate descent algorithm (CDA) $[6,12]$. The resulting algorithm sequentially updates the noiseless signals, the noise variances and other hyperparameters by analytical formulas leading to a reduced computational cost. The proposed Bayesian model and estimation algorithm are validated using synthetic and real altimetric data acquired during the Jason- 2 mission. The obtained results are very promising and show the potential of the proposed denoising strategy.

The paper is structured as follows. Section II introduces the observation model and the considered altimetric signal. The proposed hierarchical Bayesian model and its estimation algorithm are introduced in Sections III and IV. Section V validates the proposed technique using simulated data with controlled ground truth. Section VI shows results obtained using real data obtained from the Jason-2 mission. Finally, conclusions and future work are reported in Section VII.

\section{PROBLEM FORMULATION}

Consider $M$ successive signals $\boldsymbol{S} \in \mathbb{R}^{K \times M}$ and let $\boldsymbol{Y} \in$ $\mathbb{R}^{K \times M}$ denote their noisy version. Let $\boldsymbol{y}_{: m} \in \mathbb{R}^{K \times 1}$ be the $m$ th column of $\boldsymbol{Y}$ and $\boldsymbol{y}_{k \text { : }} \in \mathbb{R}^{1 \times M}$ its $k$ th row, representing the $k$ th temporal gate for all signals. For notation simplicity, we denote $\boldsymbol{y}_{: m}=\boldsymbol{y}_{m}, \forall m$, and $\boldsymbol{y}_{k:}=\boldsymbol{y}_{k}, \forall k$. Using these notations, the observation model is given by

$$
\boldsymbol{y}_{m}=\boldsymbol{s}_{m}\left(\boldsymbol{\Theta}_{m}\right)+\boldsymbol{e}_{m}, \text { with } \boldsymbol{e}_{m} \sim \mathcal{N}\left(\mathbf{0}_{K}, \boldsymbol{\Sigma}\right)
$$

where $\boldsymbol{y}_{m}$ and $s_{m}$ are $(K \times 1)$ vectors representing the $m$ th observed and noiseless signals, $\mathbf{0}_{K}$ denotes the $(K \times 1)$ vector of zeros and $\boldsymbol{e}_{m}$ is a centered Gaussian noise vector with a diagonal covariance matrix $\boldsymbol{\Sigma}=\operatorname{diag}\left(\boldsymbol{\sigma}^{2}\right)$ with $\boldsymbol{\sigma}^{2}=\left(\sigma_{1}^{2}, \cdots, \sigma_{K}^{2}\right)^{T}$ a $(K \times 1)$ vector. The signals $\boldsymbol{S}$ might depend on some parameters (by a linear or nonlinear expression) which are denoted by the $(1 \times H)$ vector $\boldsymbol{\Theta}_{m}=\left[\theta_{1}(m), \cdots, \theta_{H}(m)\right]$ containing the $H$ parameters of the $m$ th signal. Note, however, that the proposed method does not necessarily require a parametric expression for $\boldsymbol{S}$, and is valid provided that the signals satisfy some properties (as described in the following). The smooth variation of the oceanic altimetric signals (see Fig. 1 (bottom)) can be highlighted by expressing the model (1) as follows

$$
\boldsymbol{y}_{k}=\boldsymbol{s}_{k}(\boldsymbol{\Theta})+\boldsymbol{e}_{k}, \text { with } \boldsymbol{e}_{k} \sim \mathcal{N}\left(\mathbf{0}_{M}, \sigma_{k}^{2} \mathbb{I}_{M}\right)
$$

where $k \in\{1, \cdots, K\}$ indexes the signal samples that are known as "temporal gates", $\mathbb{I}_{M}$ denotes the $(M \times M)$ identity matrix and $s_{k}$ is a smooth $(M \times 1)$ vector representing the signal evolution at the $k$ th gate (see Fig. 1 (bottom) for examples). The proposed Bayesian method aims to filter the observed signals $\boldsymbol{y}_{k}, k \in\{1, \cdots, K\}$ to retrieve the noiseless signals $s_{k}, k \in\{1, \cdots, K\}$.

\subsection{Conventional altimetric model}

This section introduces the satellite altimetric model that will be considered in this paper since it satisfies the model described above. The altimetric nonlinear model accounts for three parameters $\left(P_{u}, \tau, \mathrm{SWH}\right)$ as follows $[2,9]$

$s(t)=\frac{P_{u}}{2}\left[1+\operatorname{erf}\left(\frac{t-\tau_{s}-\alpha \sigma_{c}^{2}}{\sqrt{2} \sigma_{c}}\right)\right] \exp \left[-\alpha\left(t-\tau_{s}-\frac{\alpha \sigma_{c}^{2}}{2}\right)\right]$

where $\quad \sigma_{c}^{2}=\left(\frac{\mathrm{SwH}}{2 c}\right)^{2}+\sigma_{p}^{2}, \operatorname{erf}(t)=\frac{2}{\sqrt{\pi}} \int_{0}^{t} e^{-z^{2}} d z$ is the Gaussian error function, $t$ is time, $\tau_{s}=\frac{2 \tau}{c}$ (resp. $\tau$ ) is the epoch expressed in seconds (resp. meters), $c$ is the speed of light, $\alpha$ and $\sigma_{p}^{2}$ are two known parameters (depending on the satellite and on the measurement instrument). Note that the discrete altimetric signal is gathered in the vector $s=$ $\left(s_{1}, \cdots, s_{K}\right)^{T}$, where $K=104$ gates, $s_{k}=s(k T), T$ is the time resolution and $\boldsymbol{\Theta}_{m}=\left[\mathrm{SWH}(m), \tau(m), P_{u}(m)\right]$ is a $(1 \times$ 3 ) vector containing the 3 altimetric parameters $\mathrm{SWH}, \tau, P_{u}$ for the $m$ th signal.

The altimetric signals are corrupted by speckle noise that, thanks to the averaging that takes place on-board the satellite, can be approximated by additive Gaussian noise as shown in $[6,13-15]$. Thus, the observation altimetric model satisfies (1). Moreover, the noise variances obtained, $\sigma_{k}^{2}, k \in$ $\{1, \cdots, K\}$, after the satellite averaging, are correlated due to the nature of the speckle noise (this correlation will be considered in the proposed Bayesian scheme). Finally, it is worth-noting that this paper only considers oceanic observations which generally show a smooth variation between successive signals.

\section{HIERARCHICAL BAYESIAN MODEL}

This section introduces a hierarchical Bayesian model to estimate the unknown $(K \times M)$ matrix of noiseless signals $\boldsymbol{S}$ and the $(K \times 1)$ vector $\boldsymbol{\sigma}$ containing the noise variances associated with the $M$ signals considered.

\subsection{Likelihood}

The observation model defined in (2) and the Gaussian properties of the noise sequence $\boldsymbol{e}_{k}, k \in\{1, \cdots, K\}$, yield

$$
f\left(\boldsymbol{y}_{k} \mid \boldsymbol{s}_{k}, \sigma_{k}^{2}\right) \propto\left(\frac{1}{\sigma_{k}^{2}}\right)^{\frac{M}{2}} \exp \left\{-\frac{\left\|\boldsymbol{y}_{k}-\boldsymbol{s}_{k}\right\|^{2}}{2 \sigma_{k}^{2}}\right\}
$$

where $\propto$ means "proportional to", $\|\cdot\|$ denotes the standard $l_{2}$ norm such that $\|\boldsymbol{x}\|^{2}=\boldsymbol{x}^{T} \boldsymbol{x}$ and $\boldsymbol{s}_{k}(\boldsymbol{\Theta})$ has been denoted by $s_{k}$ for brevity. Assuming independence between the observed signals leads to $f(\boldsymbol{Y} \mid \boldsymbol{\Theta}, \boldsymbol{S}) \propto \prod_{k=1}^{K} f\left(\boldsymbol{y}_{k} \mid \boldsymbol{s}_{k}, \sigma_{k}^{2}\right)$. 


\subsection{Priors for the observed signal}

As previously assumed, the successive observed signals evolve slowly leading to smooth vectors $s_{k}$, for $k \in 1, \cdots, K$ (see Fig. 1 (bottom)). This property is satisfied by considering a Gaussian prior for $s_{k}$ ensuring smoothness as follows

$$
\boldsymbol{s}_{k} \mid \epsilon_{k}^{2} \sim \mathcal{N}\left(\mathbf{0}_{M}, \epsilon_{k}^{2} \boldsymbol{H}\right),
$$

where $\boldsymbol{H}$ is an $(M \times M)$ matrix representing the squaredexponential covariance function given by $H\left(m, m^{\prime}\right)=$ $\exp \left[-\frac{\left(m-m^{\prime}\right)^{2}}{(30)^{2}}\right]$, which introduces the correlation between the successive signals and $\epsilon_{k}^{2}$ is a variance parameter that is gate dependent. From (5), it is clear that this variance is related to the energy of the signals at the $k$ th gate (via the norm $\left(\boldsymbol{s}_{k}^{T} \boldsymbol{H}^{-1} \boldsymbol{s}_{k}\right)$ ). Moreover, because of the continuity of the signal $s_{m}$ w.r.t. the temporal gates, the signal energies vary smoothly from one gate to another. Therefore, we expect $\epsilon_{k}^{2}$ to vary smoothly from one gate to another which will be introduced by considering a specific prior for $\epsilon_{k}^{2}$, as explained in Section III-D.

\subsection{Prior for the noise variance}

Due to the speckle origins of the corruption noise, we expect the noise variances $\sigma_{k}^{2}, k \in\{1, \cdots, K\}$ to vary smoothly. This behavior is considered by introducing an auxiliary vector $\boldsymbol{w}$ (of size $K \times 1$ ) and assigning a gamma Markov random field prior (GMRF) for $(\boldsymbol{\sigma}, \boldsymbol{w})$ (see $[11,16]$ for more details regarding this prior). This prior ensures that each $\sigma_{k}^{2}$ is connected to two neighboring elements of $\boldsymbol{w}$ and vice-versa via a coupling parameter $\zeta>1$ that controls the amount of correlation enforced by the GMRF (see Fig. 2 (a)). Note that the variances $\sigma_{k}^{2}, \sigma_{k^{\prime}}^{2}$ for $k \neq k^{\prime}$ are conditionally independent and that the correlation is introduced via the auxiliary variables $\boldsymbol{w}$. An interesting property of this joint prior is that the conditional prior distributions of $\boldsymbol{\sigma}$ and $\boldsymbol{w}$ reduce to conjugate inverse gamma $(\mathcal{I G})$ and gamma $(\mathcal{G})$ distributions, respectively, as follows [11]

$$
\begin{aligned}
\sigma_{k}^{2} \mid w_{k-1}, w_{k}, \zeta & \sim \mathcal{I} \mathcal{G}\left[2 \zeta, \zeta\left(w_{k-1}+w_{k}\right)\right] \\
\sigma_{K}^{2} \mid w_{K-1}, \zeta & \sim \mathcal{I} \mathcal{G}\left(\zeta, \zeta w_{K-1}\right) \\
w_{k}^{2} \mid \sigma_{k}^{2}, \sigma_{k+1}^{2}, \zeta & \sim \mathcal{G}\left[2 \zeta,\left(\frac{\zeta}{\sigma_{k}^{2}}+\frac{\zeta}{\sigma_{k+1}^{2}}\right)^{-1}\right]
\end{aligned}
$$

where $k \in\{1, \cdots, K-1\}$.

\subsection{Hyperparameter priors}

As previously explained, the hyperparameters $\epsilon_{k}^{2}$ are closely related to the energies $\left(\boldsymbol{s}_{k}^{T} \boldsymbol{H}^{-1} \boldsymbol{s}_{k}\right)$. Considering this property and the continuity of the signal suggest the presence of a correlation between the parameters $\epsilon_{k}^{2}$. This correlation can be introduced using a similar GMRF prior as in Section III-C

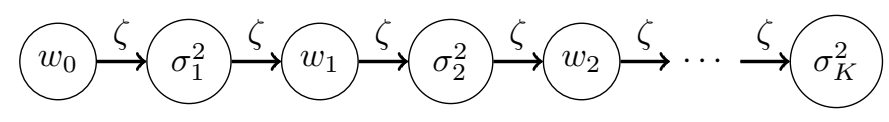

(a) GMRF for $\boldsymbol{\sigma}$

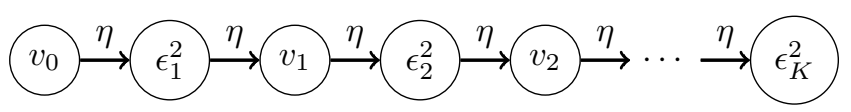

(b) GMRF for $\epsilon$

Fig. 2. Proposed 1st order GMRF neighborhood structures for (a) the noise variances $\boldsymbol{\sigma}$ and (b) the signal energies $\boldsymbol{\epsilon}$.

for the couple $(\boldsymbol{\epsilon}, \boldsymbol{v})$, where $\boldsymbol{v}$ is a new auxiliary vector and $\eta$ is the coupling parameter (see Fig. 2 (b)).

\subsection{Posterior distributions}

The parameters of interest are $\boldsymbol{X}=(\boldsymbol{S}, \boldsymbol{\sigma}, \boldsymbol{w}, \boldsymbol{\epsilon}, \boldsymbol{v})$. The joint posterior distribution of this Bayesian model can be computed using the following hierarchical structure

$$
f(\boldsymbol{X} \mid \boldsymbol{Y}) \propto f(\boldsymbol{Y} \mid \boldsymbol{S}, \boldsymbol{\sigma}) f(\boldsymbol{S} \mid \boldsymbol{\epsilon}) f(\boldsymbol{\epsilon}, \boldsymbol{v}) f(\boldsymbol{\sigma}, \boldsymbol{w}),
$$

where we have assumed a priori independence between the parameters. For simplicity, $f(x \mid \theta)$ has been denoted by $f(x)$ when the parameter $\theta$ is a user-fixed parameter.

\section{COORDINATE DESCENT ALGORITHM}

This section describes the optimization algorithm maximizing the posterior (7) w.r.t. $\boldsymbol{X}$ which provides the parameter MAP estimator. Because of the large number of parameters in $\boldsymbol{X}=(\boldsymbol{S}, \boldsymbol{\sigma}, \boldsymbol{w}, \boldsymbol{\epsilon}, \boldsymbol{v})$, we propose a coordinate descent algorithm [12] that sequentially updates the different parameters. More precisely, in each step, the posterior distribution is maximized w.r.t. one parameter, the others being fixed. This process is repeated until the algorithm has converged to a local minimum of the negative log-posterior $\mathcal{C}(\boldsymbol{S}, \boldsymbol{\sigma}, \boldsymbol{w}, \boldsymbol{\epsilon}, \boldsymbol{v})$. Thus, the algorithm iteratively updates each parameter by maximizing its conditional distribution (similarly to the iterated conditional modes algorithm [17]) which are given by

$$
\begin{aligned}
& \text { - } \boldsymbol{s}_{k} \mid \boldsymbol{y}_{k}, \sigma_{k}^{2}, \epsilon_{k}^{2} \sim \mathcal{N}\left(\overline{\boldsymbol{s}_{k}}, \boldsymbol{\Gamma}_{k}\right) \\
& \text { - } \sigma_{k}^{2} \mid \boldsymbol{y}_{k}, \boldsymbol{s}_{k}, \boldsymbol{w}_{k} \sim \mathcal{I} \mathcal{G}\left(2 \zeta+\frac{M}{2}, \frac{\beta_{1}}{2}\right) \\
& \text { - } \epsilon_{k}^{2} \mid \boldsymbol{y}_{k}, \boldsymbol{s}_{k}, \boldsymbol{v}_{k} \sim \mathcal{I} \mathcal{G}\left(2 \eta+\frac{M}{2}, \frac{\beta_{2}}{2}\right) \\
& \text { - } w_{k}^{2} \mid \sigma_{k}^{2}, \sigma_{k+1}^{2}, \zeta \sim \mathcal{G}\left[2 \zeta,\left(\frac{\zeta}{\sigma_{k}^{2}}+\frac{\zeta}{\sigma_{k+1}^{2}}\right)^{-1}\right] \\
& \text { - } v_{k}^{2} \mid \epsilon_{k}^{2}, \epsilon_{k+1}^{2}, \eta \sim \mathcal{G}\left[2 \eta,\left(\frac{\eta}{\epsilon_{k}^{2}}+\frac{\eta}{\epsilon_{k+1}^{2}}\right)^{-1}\right]
\end{aligned}
$$


where $\overline{\boldsymbol{s}_{k}}=\frac{1}{\sigma_{k}^{2}} \boldsymbol{\Gamma}_{k} \boldsymbol{y}_{k},, \boldsymbol{\Gamma}_{k}=\left(\frac{\boldsymbol{H}^{-1}}{\epsilon_{k}^{2}}+\frac{\mathbb{I}_{M}}{\sigma_{k}^{2}}\right)^{-1}, \beta_{1}=\| \boldsymbol{y}_{k}-$ $\boldsymbol{s}_{k} \|^{2}+2 \zeta\left(w_{k-1}+w_{k}\right)$ and $\beta_{2}=\boldsymbol{s}_{k}^{T} \boldsymbol{H}^{-1} \boldsymbol{s}_{k}+2 \eta\left(v_{k-1}+v_{k}\right)$. The mode of each conditional distribution is uniquely and analytically obtained which reduces the computational cost of the algorithm. In this paper, the parameters have been initialized as follows: $\boldsymbol{\sigma}^{(0)}=\boldsymbol{s}_{m}^{(0)}=\frac{1}{M} \sum_{n=1}^{M} \boldsymbol{y}_{n}, \forall m, \epsilon_{k}^{2}=10$, $\forall k$, and $w_{k}^{(0)}=v_{k}^{(0)}=10^{-12}, \forall k$. Note that more elaborate initialization procedures can be investigated, but these proposed values provided minima of good quality in the simulations considered (see Sections V and VI). More details regarding the stopping criteria of the algorithm are provided in [16].

\section{SIMULATION RESULTS ON SYNTHETIC DATA}

This section evaluates the performance of the proposed SSE (for smooth signal estimation) algorithm on synthetic data when considering two experiments. The first experiment evaluates the performance when varying the number of the denoised signals $M$. Indeed, the SSE algorithm considers successive sets of length $M$ to denoise the observed altimetric signals. Therefore, $N=5000$ signals are generated according to the altimetric model (3) while using a realistic variation of the altimetric parameters $\boldsymbol{\Theta}_{m}=\left[\operatorname{SWH}(m), \tau(m), P_{u}(m)\right]$. This realistic sequence of parameters is obtained by applying the CD-BM algorithm [6] on 5000 real Jason-2 signals (since it provides physically realistic smooth parameters) where we obtain SWH $\in[3.4,5.4]$ meters, $\tau \in[14.3,15]$ meters and $P_{u} \in[150,190]$ unit. The generated synthetic signals are then corrupted by speckle noise resulting from the averaging of $L=90$ signals and leading to $\mathrm{RSNR}=19.55 \mathrm{~dB}$. The obtained $N=5000$ signals are processed by the proposed algorithm while considering different set lengths as shown in Table I. For example, for a length set $M=250$, the algorithm is run 20 times to process the $N=5000$ signals. The performance is evaluated using the reconstruction signal to noise ratio (RSNR) given by $\mathrm{RSNR}=10 \log _{10}\left(\frac{\sum_{m=1}^{M}\left\|\boldsymbol{s}_{m}\right\|^{2}}{\sum_{m=1}^{M}\left\|\boldsymbol{s}_{m}-\widehat{\boldsymbol{s}}_{m}\right\|^{2}}\right)$, where $\boldsymbol{s}_{m}$ (resp. $\widehat{\boldsymbol{s}}_{m}$ ) denotes the noiseless (resp. denoised) signal. Overall, these results show an $\approx 11 \mathrm{~dB}$ improvement in the processed data with an increasing RSNR w.r.t. $M$. However, a high number of $M$ requires higher computational cost (mainly due to the matrix inversion in (IV)), while too small $M$ leads to more iterations. $M=500$ represents a good compromise and we consider this value for the rest of the paper [6].

The second experiment compares our algorithm with the state-of-the-art singular value decomposition (SVD) filtering strategy proposed in [7,8] (with a threshold equal to $84 \%$ ). The study is performed when varying $\mathrm{SWH} \in[0.5,8] \mathrm{m}$ with fixed $\tau=31$ gates and $P_{u}=130$. For each SWH, 500 synthetic signals are generated using the model (3) with different noise realizations ( 500 Monte carlo runs) and processed using the two considered algorithms. Table II reports the obtained RSNR when considering SVD and SSE for different SWH. This table shows an average improvement by $6 \mathrm{~dB}$ when considering SVD and by $12 \mathrm{~dB}$ when considering the proposed SSE algorithm. These results highlight the potential of the proposed strategy in denoising the altimetric signals and improving the estimation of the altimetric parameters.

\section{RESULTS ON JASON-2 REAL DATA}

This section is devoted to the validation of the proposed SSE denoising algorithm when applied to the oceanic Jason-2 dataset. The data considered cover a period of 36 minutes and consist of 43000 real signals that were extracted from pass 30 of cycle 35 . Figs. 1 (top) and (middle) present a sequence of 800 Jason- 2 signals before and after filtering. These figure clearly show a reduction in the noise affecting the signals after the application of the SSE algorithm especially in the tail of the signal (the decreasing part), which was most affected by the speckle noise. Table III shows the effect of the denoising algorithms on the estimated altimetric parameters that are obtained using the well known least-squares (LS) estimation based strategy [6,9]. This table compares the classical LS algorithm (applied to noisy data) with SVDLS and the proposed SSE-LS (LS applied to filtered data). This table shows a good agreement between the means of the estimated parameters for the LS, SVD-LS and SSE-LS algorithms (except $P_{u}$ that is slightly reduced by SSE-LS). Moreover, the smallest parameter standard-deviations (STDs) are achieved by the proposed SSE-LS. This STD reduction is of great importance for many practical applications related to oceanography such as bathymetry. Comparing SSE-LS to LS, Table III highlights an STD improvement factor by 6 for SWH, 4 for $\tau$ and 5 for $P_{u}$. Finally, Table III also compares the computational costs of the three considered algorithms when processing the 43000 signals (the result is reported for each signal). Because of the filtering step, both SVD-LS and SSE-LS require more computational time than LS. Note that the proposed SSE algorithm requires more computational time than the SVD approach. However, this cost (about $12 \%$ of additional computational times w.r.t. the LS algorithm) must be balanced by the performance improvement in terms of RSNR and parameter STDs. These results confirm the good performance of the proposed strategy for denoising smooth signals such as oceanic altimetric signals.

\section{CONCLUSIONS}

This paper has presented a new Bayesian strategy for denoising smooth signals corrupted by Gaussian noise. The method assumed a reduced evolution of successive continuous signals, which can have a numerical expression or be given by a linear/nonlinear function with respect to some parameters. A Bayesian model was proposed to take into 
Table 1. Performance of the proposed SSE algorithm w.r.t. the filter length (5000 signals). The corrupted data presents an $\mathrm{RSNR}=19.55 \mathrm{~dB}$.

\begin{tabular}{|c|c|c|c|c|c|c|c|}
\cline { 2 - 8 } \multicolumn{1}{c|}{} & \multicolumn{7}{c|}{ Filter length } \\
\cline { 2 - 8 } \multicolumn{1}{c|}{} & 50 & 100 & 250 & 500 & 1000 & 2500 & 5000 \\
\hline RSNR (dB) & 31.1 & 31.4 & 31.5 & 31.6 & 31.7 & 31.7 & 31.7 \\
\hline Time per signal (ms) & 0.35 & 0.24 & 0.25 & 0.38 & 1.03 & 5.00 & 17.47 \\
\hline
\end{tabular}

Table 2. RSNR (in dB) with respect to SWH. The corrupted data presents an RSNR $=19.55 \mathrm{~dB}$.

\begin{tabular}{|c|c|c|c|c|c|c|c|c|c|c|}
\cline { 3 - 12 } \multicolumn{2}{c|}{} & \multicolumn{10}{c|}{ SWH $(\mathrm{m})$} \\
\cline { 2 - 12 } \multicolumn{2}{c|}{} & 0.5 & 1 & 2 & 3 & 4 & 5 & 6 & 7 & 8 \\
\hline \multirow{2}{*}{ RSNR (dB) } & SVD & 26.35 & 26.43 & 26.30 & 26.02 & 26.03 & 26.07 & 26.08 & 25.92 & 25.86 \\
\cline { 2 - 11 } & SSE & 32.24 & 32.21 & 32.22 & 32.13 & 32.15 & 32.10 & 32.22 & 32.13 & 32.07 \\
\hline
\end{tabular}

Table 3. Performance on real Jason-2 data (45000 signals).

\begin{tabular}{|c|c|c|c|c|}
\cline { 3 - 5 } \multicolumn{2}{c|}{} & SWH $(\mathrm{cm})$ & $\tau(\mathrm{cm})$ & $P_{u}$ \\
\hline \multirow{3}{*}{ Mean } & LS & 242 & 14.68 & 167.73 \\
\cline { 2 - 5 } & SVD-LS & 241 & 14.67 & 166.62 \\
\cline { 2 - 5 } & SSE-LS & 248 & 14.68 & 164.83 \\
\hline \hline \multirow{3}{*}{ STD } & LS & 59.9 & 12.01 & 6.18 \\
\cline { 2 - 5 } & SVD-LS & 18.14 & 6.02 & 6.09 \\
\cline { 2 - 5 } & SSE-LS & $\mathbf{9 . 0 3}$ & $\mathbf{2 . 9 4}$ & $\mathbf{1 . 2 1}$ \\
\hline \hline \multirow{2}{*}{$\begin{array}{c}\text { Average time } \\
\text { per signal (ms) }\end{array}$} & LS & \multicolumn{3}{|c|}{$\mathbf{8 . 5 6}$} \\
\cline { 2 - 5 } & SVD-LS & \multicolumn{3}{|c}{9.05} \\
\cline { 2 - 5 } & SSE-LS & \multicolumn{3}{|c}{9.63} \\
\hline
\end{tabular}

account the Gaussian properties of the noise, and the smooth properties of the signal evolution. The resulting posterior distribution was maximized using a fast coordinate descent algorithm that showed good results on both synthetic and real altimetric signals. The proposed algorithm was also evaluated by combining it with a commonly used parameter estimation strategy for the altimetric parameters. The estimated parameters showed a clear improvement highlighting the benefit of the proposed algorithm. It is worth-noting that the proposed strategy is fast and generic and thus could be applied when considering other altimetric technologies such as delay/Doppler altimetry $[10,18,19]$. This point will be considered in future work.

\section{REFERENCES}

[1] B. W. Hapke, "Bidirectional reflectance spectroscopy. I. Theory," $J$. Geophys. Res., vol. 86, pp. 3039-3054, 1981.

[2] G. Brown, "The average impulse response of a rough surface and its applications," IEEE Trans. Antennas and Propagation, vol. 25, no. 1, pp. 67-74, Jan. 1977.

[3] A. Halimi, C. Mailhes, J.-Y. Tourneret, P. Thibaut, and F. Boy, "Parameter estimation for peaky altimetric waveforms," IEEE Trans. Geosci. Remote Sens., vol. 51, no. 3, pp. 1568-1577, March 2013.

[4] S. Maus, C. M. Green, and J. D. Fairhead, "Improved ocean-geoid resolution from retracked ERS-1 satellite altimeter waveforms," Geophysical Journal International, vol. 134, no. 1, pp. 243-253, Feb. 1998.
[5] D. T. Sandwell and W. H. F. Smith, "Retracking ERS-1 altimeter waveforms for optimal gravity field recovery," Geophys. J. Int., vol. 163, no. 1, pp. 79-89, Oct. 2005.

[6] A. Halimi, C. Mailhes, J.-Y. Tourneret, and H. Snoussi, "Bayesian estimation of smooth altimetric parameters: Application to conventional and delay/Doppler altimetry," IEEE Trans. Geosci. Remote Sens., 2015, to appear.

[7] A. Ollivier, "Nouvelle approche pour l'extraction de paramètres géophysiques à partir des mesures en altimétrie radar," Ph.D. dissertation, Institut National Polytechnique de Grenoble, Grenoble, France, 2006.

[8] P. Thibaut, J. Poisson, A. Ollivier, E. Bronner, and N. Picot, "Singular value decomposition applied on altimeter waveforms," in Report of the Ocean Surf. Topogr. Sci. Team Meet. (OSTST), Seattle, USA, June 2009. [Online]. Available: http://www.aviso.altimetry.fr/ fileadmin/documents/OSTST/2009/oral/Thibaut_SVD2.pdf

[9] L. Amarouche, P. Thibaut, O. Z. Zanife, J.-P. Dumont, P. Vincent, and N. Steunou, "Improving the Jason-1 ground retracking to better account for attitude effects," Marine Geodesy, vol. 27, no. 1-2, pp. 171-197, Aug. 2004.

[10] A. Halimi, C. Mailhes, J.-Y. Tourneret, P. Thibaut, and F. Boy, "A semianalytical model for delay/Doppler altimetry and its estimation algorithm," IEEE Trans. Geosci. Remote Sens., vol. 52, no. 7, pp. 42484258, July 2014.

[11] O. Dikmen and A. Cemgil, "Gamma markov random fields for audio source modeling," IEEE Trans. Audio, Speech, Language Process., vol. 18, no. 3, pp. 589-601, March 2010.

[12] D. P. Bertsekas, Nonlinear programming. Belmont, Massachusetts: Athena Scientific, 1995.

[13] C. Martin-Puig and G. Ruffini, "SAR altimeter retracker performance bound over water surfaces," in Proc. IEEE Int. Conf. Geosci. Remote Sens. (IGARSS), Cape Town, South Africa, July 12-17, 2009, pp. 449 -452 .

[14] O. Germain and G. Ruffini, "A revisit to the GNSS-R code range precision," in Proc. GNSS-R, Noordwijk, The Netherlands, June 14-15, 2006.

[15] A. Halimi, C. Mailhes, and J.-Y. Tourneret, "Cramér-Rao bounds and estimation algorithms for delay/Doppler and conventional altimetry," in Proc. EUSIPCO, Marrakech-Marocco, Sept. 9-13 2013.

[16] A. Halimi, G. Buller, S. McLaughlin, and P. Honeine, "Bayesian filtering of smooth signals: Application to altimetry," in ArXiv e-prints, Feb. 2016.

[17] J. Besag, "On the statistical analysis of dirty pictures," Journal of the Royal Statistical Society, Series B, vol. 48, no. 3, pp. 48-259, 1986.

[18] R. K. Raney, "The delay/Doppler radar altimeter," IEEE Trans. Geosci. Remote Sens., vol. 36, no. 5, pp. 1578-1588, Sept. 1998.

[19] A. Halimi, C. Mailhes, J.-Y. Tourneret, T. Moreau, and F. Boy, "Including antenna mispointing in a semi-analytical model for delay/Doppler altimetry," IEEE Trans. Geosci. Remote Sens., vol. 53, no. 2, pp. 598608, Feb. 2015 